Article

\title{
Neural Network Based AMP Method for Multi-User Detection in Massive Machine-Type Communication
}

\author{
Mengjiang Sun ${ }^{1}$ and Peng Chen ${ }^{2, *}$ \\ 1 School of Information Science and Engineering, Southeast University, Nanjing 211189, China; \\ 213170963@seu.edu.cn \\ 2 State Key Laboratory of Millimeter Waves, Southeast University, Nanjing 210096, China \\ * Correspondence: chenpengseu@seu.edu.cn
}

Received: 8 July 2020; Accepted: 10 August 2020; Published: 11 August 2020

\begin{abstract}
In massive machine-type communications (mMTC) scenarios, grant-free non-orthogonal multiple access becomes crucial due to the small transmission latency, limited signaling overhead and the ability to support massive connectivity. In a multi-user detection (MUD) problem, the base station (BS) is unaware of the active users and needs to detect active devices. With sporadic devices transmitting signals at any moment, the MUD problem can be formulated as a multiple measurement vector (MMV) sparse recovery problem. Through the Khatri-Rao product, we prove that the MMV problem is transformed into a single measurement vector (SMV) problem. Based on the basis pursuit de-noising approximate message passing (BPDN-AMP) algorithm, a novel learning AMP network (LAMPnet) algorithm is proposed, which is designed to reduce the false alarm probability when the required detection probability is high. Simulation results show that when the required detection probablity is high, the AMP algorithm based on LAMPnet noticeably outperforms the traditional algorithms with acceptable computational complexity.
\end{abstract}

Keywords: massive machine-type communication; multi-user detection; approximate message passing; sparse recovery

\section{Introduction}

With the rapid growth in the number of wireless connected machines, massive machine-type communications (mMTC) [1,2] have been listed as one of the three main use cases in the fifth-generation (5G) cellular technologies road map [3]. The current grant based method for uplink transmission in the fourth-generation system requires a procedure to ask for the grant of the base station (BS), bringing attendant large transmission latency and excessive signaling overhead [4]. The mechanism disables grant based access from being adopted in the $5 \mathrm{G}$ system. Consequently, a grant-free non-orthogonal multiple access is proposed [5-7]. However, the BS is unaware of the active users without the granting process and needs to detect active devices among large number of potential devices, which is named multi-user detection (MUD) [8].

Multi-user detector includes an optimal detector and a sub-optimal detector. An optimal detector is actually a maximum likelihood sequence detector and can only be used with amplitudes and phases of all users as prior information. Besides, exponential complexity of an optimal detector prevents it from practical application [9]. A sub-optimal detector includes a linear detector and a nonlinear detector $[10,11]$. A linear detector uses a linear transformation on the output of the correlator before making a judgement. Decorrelating linear MUD and minimum mean square MUD are typical linear detectors. A nonlinear detector mainly focuses on interference cancellation. Parallel interference cancellers and serial interference cancellers are typical nonlinear detectors. 
A crucial feature of mMTC is that only a small fraction of the potentially devices are active at any moment, thus MUD problem can be formulated as a sparse recovery problem [12]. Studies have been made to address MUD problem with compressed sensing (CS) in the literature. Orthogonal matching pursuit (OMP) [13-15] is widely used and modified in the field. In [16], a greedy algorithm based on OMP for MUD is proposed. A dynamic active user detection based on OMP is proposed in [17] to realize both user activity and data detection in several continuous time slots. For the BS with multiple antennas, OMP for multiple measurement vector (MMV) is proposed and analyzed in [18]. However, the computational complexity of OMP based algorithms increases rapidly when more active users are connected. Comparatively, computationally more efficient approximate message passing (AMP) algorithm is used for MUD in [19-21].

AMP is proposed by Donoho and others $[22,23]$ and is a simplification of belief propagation in effect. When applied in different circumstances, AMP transforms to different types [23]. Basis pursuit AMP (BP-AMP) and basis pursuit de-noising AMP (BPDN-AMP) [24] are designed for basis pursuit problem like MUD. Assuming that the distribution of original signal is unknown, BP-AMP and BPDN-AMP adopt soft threshold denoiser. BP-AMP is a special case of BPDN-AMP without noise and they differ in the updating to the threshold. Thouless-Anderson-Palmer AMP (TAP-AMP) and Bayesian optimal AMP (BAMP) [25] are deduced with distribution of original signal as prior information and the denoiser is designed based on Bayesian methodology. The main idea of AMP is to simplify the belief propagation process with central limit theorem and Taylor expansion. Through assuming the messages obey Gaussian distribution, the computational complexity is greatly reduced.

In this paper, since AMP can only be applied in single measurement vector (SMV) problem, we formulate the original MMV problem as a SMV problem through Khatri-Rao product. Besides, the learning AMP network (LAMPnet) is proposed to reduce the false alarm probability and optimize the detection performance, especially when a high detection probablity is required. The LAMPnet is designed to study the updating of the threshold in BPDN-AMP and the LAMPnet-AMP returns the detected active users together with their reliabilities. Through setting a threshold of the realibility, the active users can be ultimately determined.

The rest of the paper is organized as follows. In Section 2, we establish uplink grant-free transmission system model and convert the MUD problem into a SMV problem through Khatri-Rao product. In Section 3, AMP is introduced. Then the proposed algorithm LAMPnet is shown in Section 4. Section 5 is devoted to comparison among LAMPnet-AMP, BPDN-AMP and MMV-OMP. The conclusion is drawn in Section 6. Notations of symbols are shown in Table 1.

Table 1. Notations.

\begin{tabular}{cc}
\hline Symbol & Quantity \\
\hline$M$ & number of antennas at the base station \\
$N$ & number of potentially active users \\
$L$ & length of the pilot sequence \\
$D$ & number of LAMPnets used in LAMPnet-AMP \\
$K$ & number of active users \\
$P$ & iterations threshold of LAMPnet-AMP \\
$T_{B P D N-A M P}$ & number of iterations in BPDN-AMP \\
$T_{L A M P n e t-A M P}$ & number of iterations in LAMPnet-AMP \\
$T_{\text {OMP-MMV }}$ & number of iterations in OMP-MMV \\
\hline
\end{tabular}




\section{System Model}

Consider an uplink grant-free transmission system with a BS and $N$ potentially active devices (see Figure 1). Assume that the BS possess $M$ antennas for signal transmission and reception. Then the received signal at the $i$-th antenna is

$$
\mathbf{y}_{i}=\sum_{u=1}^{N} h_{u i} \alpha_{u} \mathbf{s}_{u}+\mathbf{w} \triangleq \mathbf{S x}_{i}+\mathbf{w}_{i}
$$

where $\mathbf{y}_{i} \in \mathbb{C}^{L \times 1}$ is the received signal at the $i$-th antenna and $\alpha_{u} \in\{1,0\}$ represents whether the $u$-th device is active or not. $h_{u i}$ is the fading channel gain between the $u$-th device and the $i$-th antenna, with the channel assumed to be a Rayleigh flat channel. $\mathbf{s}_{u} \in \mathbb{R}^{L \times 1}$ is denoted as the pilot sequence for the $u$-th device, which is unique in order to distinguish different users. $\mathbf{w}_{i} \in \mathbb{C}^{L \times 1}$ is an additive white Gaussian noise. $\mathbf{S} \triangleq\left[\mathbf{s}_{1}, \mathbf{s}_{2} \cdots \mathbf{s}_{N}\right] \in \mathbb{R}^{L \times N}, \mathbf{x}_{i} \triangleq\left[\mathbf{x}_{1 i}, \mathbf{x}_{2 i} \cdots \mathbf{x}_{N i}\right]^{T} \in \mathbb{C}^{N \times 1}$ and $\mathbf{x}_{u i} \triangleq h_{u i} \alpha_{u}$. Then the model can be built as

$$
\mathbf{y}=\sum_{u=1}^{N} h_{u i} \alpha_{u} \mathbf{s}_{u}+\mathbf{w} \triangleq \mathbf{S} \mathbf{x}+\mathbf{w}
$$

where $\mathbf{y} \triangleq\left[\mathbf{y}_{1}, \mathbf{y}_{2} \cdots \mathbf{y}_{M}\right] \in \mathbb{C}^{L \times M}, \mathbf{x} \triangleq\left[\mathbf{x}_{1}, \mathbf{x}_{2} \cdots \mathbf{x}_{M}\right] \in \mathbb{C}^{N \times M}, \mathbf{w} \triangleq\left[\mathbf{w}_{1}, \mathbf{w}_{2} \cdots \mathbf{w}_{M}\right] \in \mathbb{C}^{L \times M}$.

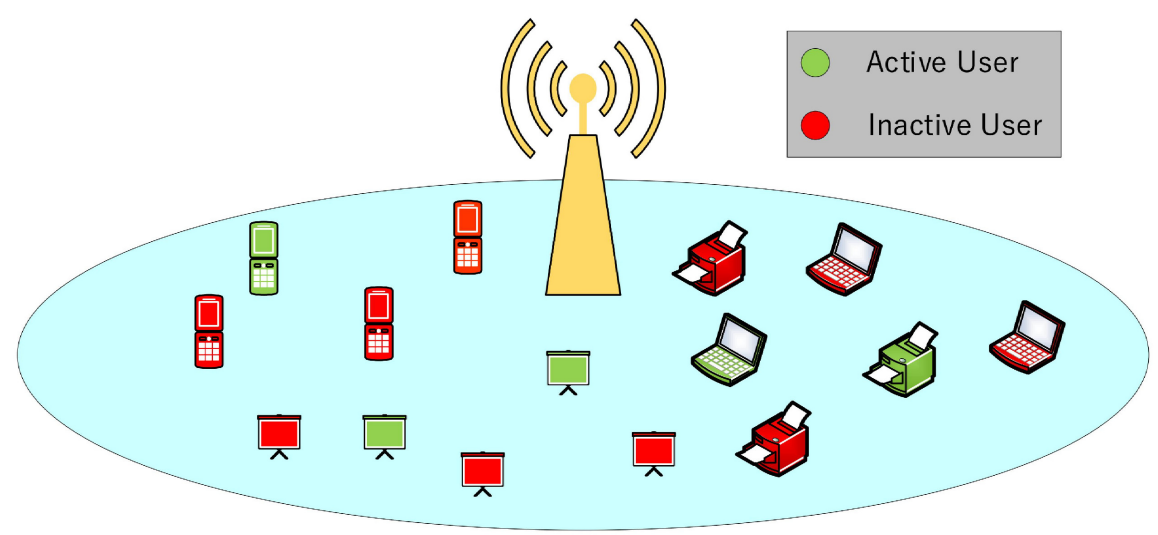

Figure 1. Uplink grant-free transmission system with a base station (BS) and massive devices.

When the model is built under a multipath channel, slight revisions need to be made. Considering that the time delays in different paths differ, a cyclic shift matrix $\tilde{\mathbf{S}_{k}}$ is defined as

$$
\tilde{\mathbf{s}_{k}}=\left[\begin{array}{cccc}
\mathbf{s}_{k, 0} & \mathbf{s}_{k, L-1} & \cdots & \mathbf{s}_{k, 1} \\
\mathbf{s}_{k, 1} & \mathbf{s}_{k, 0} & \cdots & \mathbf{s}_{k, 2} \\
\cdots & \cdots & \cdots & \cdots \\
\mathbf{s}_{k, L-1} & \mathbf{s}_{k, L-2} & \cdots & \mathbf{s}_{k, 0}
\end{array}\right],
$$

where $\tilde{\mathbf{S}_{k}} \in \mathbb{R}^{L \times L}$. Rows in $\tilde{\mathbf{S}_{k}}$ represent the pilot sequence of user $k$ with different time delays. Then the received signal at the $i$-th antenna is

$$
\mathbf{y}_{i}=\sum_{k=1}^{N} \tilde{\mathbf{S}_{k}} \mathbf{u}_{i k}+\mathbf{w}_{i}=\tilde{\mathbf{S}} \mathbf{u}_{i}+\mathbf{w}_{i}
$$

$\mathbf{y}_{i} \in \mathbb{C}^{L \times 1}$ is the received signal at the $i$-th antenna. $\mathbf{u}_{i k} \in \mathbb{C}^{L \times 1}$ is the channel gain between the $k$-th device and the $i$-th antenna and the entries in $\mathbf{u}_{i k}$ correspond to different time delays. $\tilde{\mathbf{S}} \triangleq\left[\tilde{\mathbf{S}_{1}}, \tilde{\mathbf{S}_{2}} \cdots \tilde{\mathbf{S}_{N}}\right] \in \mathbb{R}^{L \times L N}, \mathbf{u}_{i} \triangleq\left[\mathbf{u}_{i 1}^{T}, \mathbf{u}_{i 2}^{T} \cdots \mathbf{u}_{i N}^{T}\right]^{T} \in \mathbb{C}^{L N \times 1}$. The complete multipath channel model can be built as 


$$
\mathbf{y}=\tilde{\mathbf{S}} \mathbf{u}+\mathbf{w},
$$

where $\mathbf{y} \triangleq\left[\mathbf{y}_{1}, \mathbf{y}_{2} \cdots \mathbf{y}_{M}\right] \in \mathbb{C}^{L \times M}, \mathbf{u} \triangleq\left[\mathbf{u}_{1}, \mathbf{u}_{2} \cdots \mathbf{u}_{M}\right] \in \mathbb{C}^{L N \times M}$. Obviously, the difference between the model under multipath channel and the model under single path channel is mainly about the size of the sparse signal. However, the difference in size is negligible in the following process. Thus we adopt the model under single path channel in the rest of this paper.

The MUD problem aims at detecting all the $K$ active users $u_{1}, u_{2} \cdots u_{K}$ with $\alpha_{u j}=1, j=1,2 \cdots K$, i.e., identifying indices of the $K$ non-zero rows in $\mathbf{x}$. The length of pilot sequence $L$ is much smaller than the number of devices $N$ due to the limited coherence time in a practical mMTC scenario. Additionally, number of the non-zero rows in $\mathbf{x}$ is small since only sporadic devices transmit signals at any moment. Therefore, the MUD problem is viewed as a MMV problem of CS [26].

Theoretically, the self covariance matrix of $\mathbf{y}_{i}$ is

$$
\mathbf{R}=E\left(\mathbf{y}_{i} \mathbf{y}_{i}^{H}\right)=\mathbf{S}\left(\mathbf{x}_{i} \mathbf{x}_{i}^{H}\right) \mathbf{S}^{H}+\sigma_{z}^{2} \mathbf{I}_{L}=\mathbf{S} \boldsymbol{\Lambda} \mathbf{S}^{H}+\sigma_{z}^{2} \mathbf{I}_{L} .
$$

By applying Khatri-Rao product, it can be formulated as the following SMV problem.

$$
\operatorname{vec}(\mathbf{R})=\left(\mathbf{S}^{*} \odot \mathbf{S}\right) \cdot \operatorname{diag}(\mathbf{\Lambda})+\operatorname{vec}\left(\sigma_{z}^{2} \mathbf{I}_{L}\right)
$$

$\mathbf{S}^{*}$ is the conjugate matrix of $\mathbf{S}$. $\mathbf{R}$ can be estimated based on the received signals in $M$ antennas.

$$
\hat{\mathbf{R}}=\frac{1}{M} \sum_{i=1}^{M} \mathbf{y}_{i} \mathbf{y}_{i}^{H}
$$

Since $\left(\mathbf{x}_{i} \mathbf{x}_{i}^{H}\right)$ and $\mathbf{S}$ are both real matrixes, $\mathbf{R}$ is a real matrix as well. Thus the imagine part in $\hat{R}$, which is generated due to errors, should be ignored. Define $\mathbf{Y} \triangleq \operatorname{real}\left(\operatorname{vec}\left(\frac{1}{M} \sum_{i=1}^{M} \mathbf{y}_{i} \mathbf{y}_{i}^{H}\right)\right), \boldsymbol{\Phi} \triangleq \mathbf{S}^{*} \odot \mathbf{S}$ and the MUD problem is reformulated as

$$
\mathbf{Y}=\mathbf{\Phi} \mathbf{x}+\mathbf{n}
$$

The non-zero entries in $\mathbf{x}$ represnet the active users and the target is to estimate $\mathbf{x}$ from $\mathbf{Y}$. The estimation of $\mathbf{x}$ is equivalent to solving an underdetermined equation. It will be hard to obtain an accurate solution without using sparse property of $\mathbf{x}$. Thus, an $l_{0}$-norm minimization can be applied as

$$
\min \|\mathbf{x}\|_{0}, \mathbf{Y}=\mathbf{\Phi} \mathbf{x} .
$$

The optimization is a $l_{0}$ problem, a classical NP hard problem. If $\boldsymbol{\Phi}$ meets restricted isometry property (RIP), it can be transferred to $l_{1}$ problem. RIP is defined as follows.

For each integer $s=1,2, \ldots$, define the isometry constant $\delta_{s}$ of a matrix $\Phi$ as the smallest number such that

$$
\left(1-\delta_{s}\right)\|\mathbf{x}\|_{2}^{2} \leq\|\mathbf{\Phi x}\|_{2}^{2} \leq\left(1+\delta_{s}\right)\|\mathbf{x}\|_{2}^{2}
$$

holds for all $s$-sparse vectors. Candès also explains the relation between $\delta_{2 s}$ and the solution of the $l_{0}$-norm minimization problem [27]:

- If $\delta_{2 s}<1$, the $l_{0}$ problem has a unique s-sparse solution;

- If $\delta_{2 s}<\sqrt{2}-1$,the solution of the $l_{1}$ problem is that of the $l_{0}$ problem.

A matrix $\mathbf{S}$ with $\delta_{2 s}<1$ guarantees that any s-sparse solution is unique. Actually, any $2 s$ columns in $\Phi$ are linearly independent when $\delta_{2 s}<1$, which means that it is impossible for different s-sparse signals to be mapped to one observation signal y. When $\delta_{2 s}<\sqrt{2}-1$, it can be ensured that the solution to $l_{1}$ problem is that of $l_{0}$ problem. The conclusion is important because $l_{0}$ problem can be solved by convex optimization and many sparse signal recovery algorithms are base on it. Tao and Candès also prove that a normal random matrix can be used as observation matrix [28]. In our paper, 
the pilots are generated from Gaussian distributions with zero mean and variance $\frac{1}{L}$, thus $\boldsymbol{\Phi}$ meets RIP and we only need to solve a $l_{1}$ problem instead

$$
\min \|\mathbf{x}\|_{1}, \mathbf{Y}=\mathbf{\Phi} \mathbf{x}
$$

As mentioned ahead, BP-AMP and BPDN-AMP are both designed for basis pursuit problem and we will introduce them in the next part. Another kind of recovery algorithms like OMP are greedy algorithms, and are basically realized by iterations. In each iteration, one column in the over-complete dictionary matrix is selected as the one most related to the current residuals. Least mean square (LMS) method is used to recover sparse signals based on the columns selected in previous iterations, and residuals will be updated afterwards. When residuals are smaller than a threshold or the number of non-zero values in recovered signal is equal to sparsity, it stops iteration.

\section{AMP for MUD}

AMP and MP are both proposed based on belief propagation and iteration threshold method. MP algorithm updates all the signals between nodes in factor graph, which makes it complicated, and AMP algorithm takes approximations using central limit theorem and Taylor expansion to reduce the complexity. The general form of AMP is as follows.

$$
\begin{gathered}
\mathbf{x}^{t+1}=\eta\left(\mathbf{x}^{t}+\boldsymbol{\Phi}^{T} \mathbf{z}^{t} ; \lambda\right), \\
\mathbf{z}^{t+1}=\mathbf{Y}-\boldsymbol{\Phi} \mathbf{x}^{t}+\frac{1}{\delta}\left\langle\eta^{\prime}\left(\mathbf{x}^{t}+\boldsymbol{\Phi}^{T} \mathbf{z}^{t} ; \lambda\right)\right\rangle,
\end{gathered}
$$

where $\eta$ is the denoiser function and $\eta^{\prime}$ is its first order derivative. $\mathbf{x}^{t}$ is the recovered signal in $t$-th generation. $\mathbf{z}^{t}$ is the residual in $t$-th generation. $\lambda$ is the threshold level. $\delta$ is the ratio of the number of rows to the number of columns of $\boldsymbol{\Phi}$. Different types of AMP algorithms mainly differ in the design of the denoiser $\eta$ [29-31].

When AMP is used for basis pursuit problem, the soft threshold function $\eta_{s}$ is used as the denoiser, which is defined as

$$
\eta_{s}(x ; b)=\left\{\begin{array}{cl}
x-b & (x>b) \\
0 & (|x| \leqslant b) . \\
x+b & (x<-b)
\end{array}\right.
$$

Since noise exists in transmission, BPDN-AMP is adopted here, which can be expressed as follows.

$$
\begin{gathered}
\mathbf{x}^{t+1}=\eta_{s}\left(\mathbf{x}^{t}+\boldsymbol{\Phi}^{T} \mathbf{z}^{t} ; \lambda+\gamma^{t}\right), \\
\mathbf{z}^{t+1}=\mathbf{Y}-\boldsymbol{\Phi} \mathbf{x}^{t}+\frac{1}{\delta}\left\langle\eta_{s}\left(\mathbf{x}^{t}+\boldsymbol{\Phi}^{T} \mathbf{z}^{t} ; \lambda+\gamma^{t}\right)\right\rangle, \\
\gamma^{t+1}=\frac{\lambda+\gamma^{t}}{\delta}\left\langle\eta_{s^{\prime}}\left(\mathbf{x}^{t}+\boldsymbol{\Phi}^{T} \mathbf{z}^{t} ; \lambda+\gamma^{t}\right)\right\rangle,
\end{gathered}
$$

$\gamma^{t}$ is the threshold level in $t$-th generation and $\lambda$ is the original threshold level. AMP iteratively produces a signal with noise $\mathbf{x}^{t}+\boldsymbol{\Phi}^{T} \mathbf{z}^{t}$ and uses the denoiser to minimize the mean square error (MSE). Device $n$ is declared to be active if $\mathbf{x}_{n}$ in the output is non-zero and declared to be inactive otherwise.

\section{AMP Based on LAMPnet for MUD}

The LAMPnet is proposed to study the updating of the threshold level in BPDN-AMP and LAMPnet based AMP uses LAMPnet to update the threshold in each iteration. After training $D$ LAMPnets, AMP based on the $D$ LAMPnets totally returns $D$ sets of detected users. When different neural networks are trained with the same training set, they are still not all the same due to chances in the training process such as initialization of weights and thresholds, especially when the structure of 
network is simple. Thus the $D$ sets of users are similar but different. The frequency of an user being declared to be active in all the $D$ sets represents the reliability of its activation. Through setting a frequency threshold, reliable active users can be detected ultimately.

\subsection{Studying the Update to Threshold Level}

In each iteration of AMP algorithm, the update to $\gamma$ is a polynomial which contains $\eta^{\prime}\left(\mathbf{x}^{t}+\boldsymbol{\Phi}^{T} \mathbf{z}^{t} ; \lambda+\gamma^{t}\right)$. Such update is possible to be studied by neural network and the result confirms it. Training a neural network to study the update to $\gamma$ and using it to update $\gamma$ instead of $\gamma^{t+1}=\frac{\lambda+\gamma^{t}}{\delta}\left\langle\eta^{\prime}\left(\mathbf{x}^{t}+\boldsymbol{\Phi}^{T} \mathbf{z}^{t} ; \lambda+\gamma^{t}\right)\right\rangle$ proves to recover signals successfully with its RMSE close to the result of AMP. Besides, when different neural networks with the same structure, inputs and outputs are trained to complete the recovery, outputs of them have slight differences as expected. Such differences are obvious when structure of the network is simple. Figure 2 shows the algorithm of the back propagation networks used in this paper.

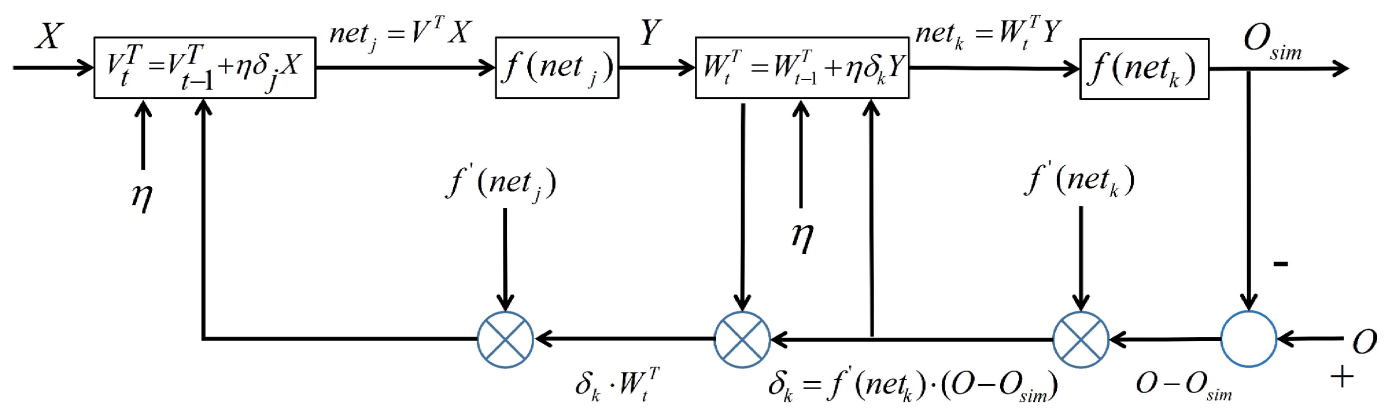

Figure 2. Algorithm of the proposed neural network.

The input of the neural network is $\mathbf{x}^{t}+\boldsymbol{\Phi}^{T} \mathbf{z}^{t}$ in each iteration of BPDN-AMP and the expected output of the neural network is the corresponding threshold $\gamma^{t+1}$. Through recording $\mathbf{x}^{t}+\boldsymbol{\Phi}^{T} \mathbf{z}^{t}$ and $\gamma^{t+1}$ in iterations of BPDN-AMP, adequate samples can be obtained as training dataset. With only 1 hidden layer and 6 neurons in the hidden layer, the network is trained quite quickly. Linear transfer function is used as activation function for output layer and Tan-sigmoid function is used for hidden layer. Mean square error is used as loss function. Figure 3 shows the training phase, validation phase and test phase of a LAMPnet. After 21 epochs, it reaches best validation performance.

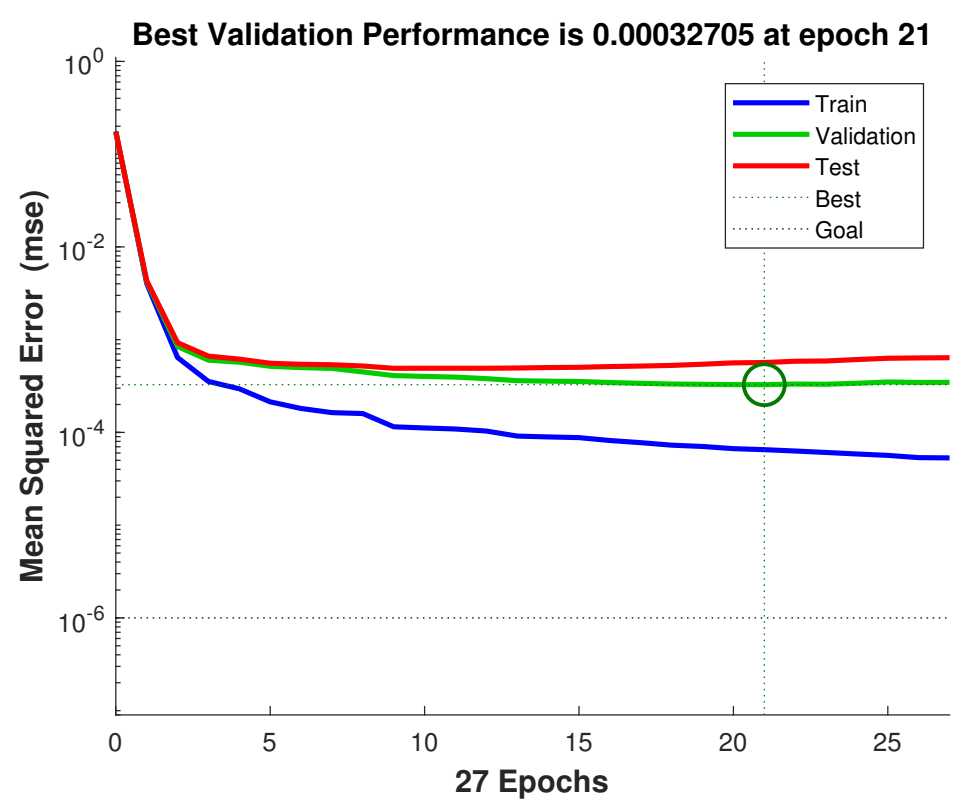

Figure 3. Training process of the proposed neural network. 


\subsection{Detecting Active Users}

When $D$ different LAMPnets are used, $D$ signals will be recovered. In order to eliminate the mistakenly detected users, we adopt a hard threshold function $\eta_{h}$ with an adaptive threshold to filter the small values in the recovered signals.

$$
\eta_{h}\left(\mathbf{x}_{n} ; b\right)=\left\{\begin{array}{cc}
\mathbf{x}_{n} & \left(\left|\mathbf{x}_{n}\right|>b\right) \\
0 & \left(\left|\mathbf{x}_{n}\right| \leqslant b\right)
\end{array},\right.
$$

where $b$ is defined as the average absolute value of the non-zero entries in the recovered $\mathbf{x}$. While the filtering causes missed detection, the losses can be corrected by using more different LAMPnets. The output of the $D$ recovered signals converts to $D$ sets of estimated active users. Reliability of user activation can be expressed by the frequency of users appearing in the sets. Through setting a frequency threshold $\theta$, the users whose appearance frequency are over $\theta$ become the ultimately determined active users. The detailed algorithm of AMP-NN is listed in Algorithm 1.

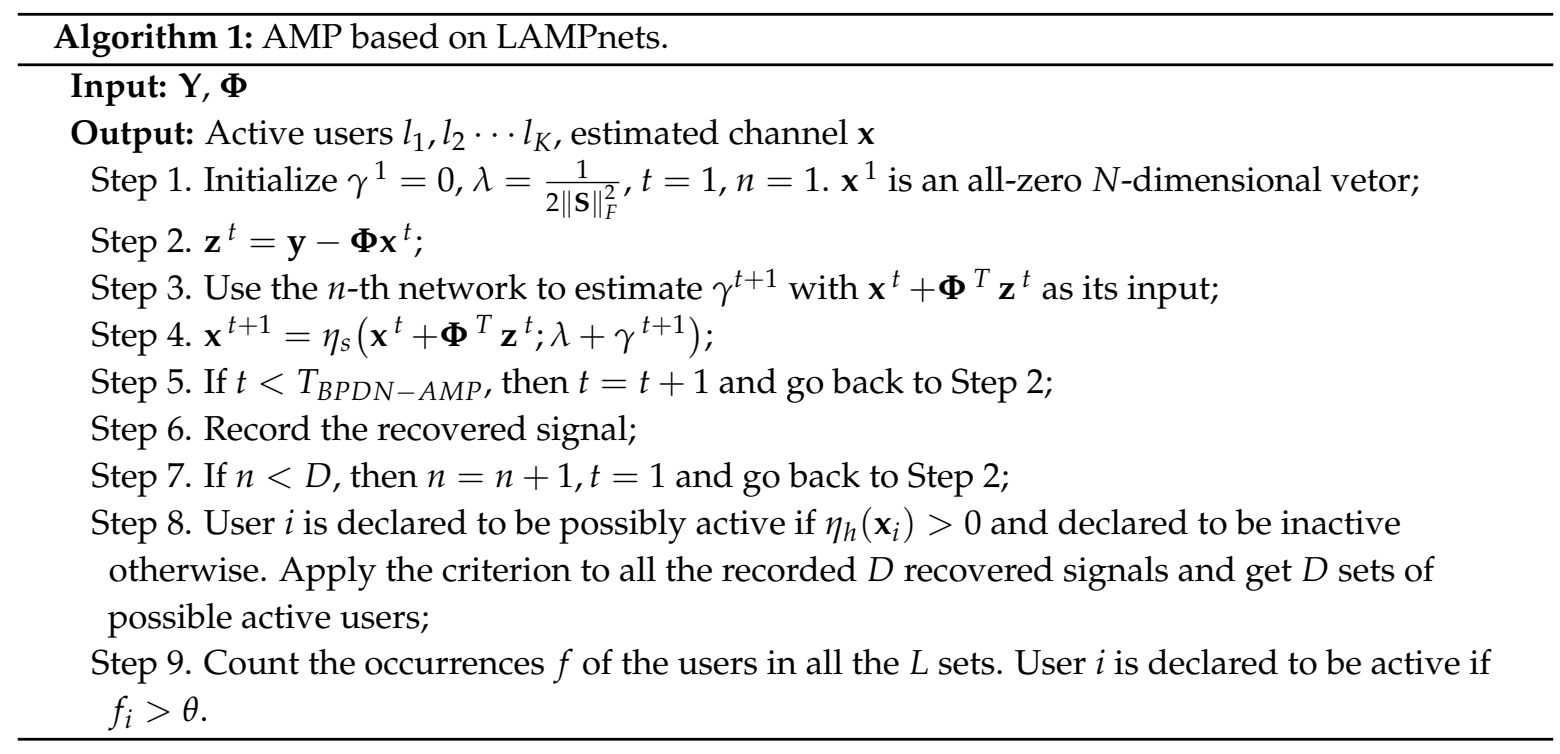

The computational complexity of KR-BPDN-AMP, OMP-MMV and KR-LAMPnet-AMP are shown in Table 2.

Table 2. Computational complexity.

\begin{tabular}{cc}
\hline KR-BPDN-AMP & $O\left(2 N L^{2} T_{B P D N-A M P}\right)$ \\
KR-LAMPnet-AMP & $O\left(2 N L^{2} D T_{L A M P n e t-A M P}\right)$ \\
OMP-MMV & $O\left(\left(N^{2} L^{2}+N^{3}\right) T_{O M P-M M V}\right)$ \\
\hline
\end{tabular}

\subsection{Channel Estimation}

Additionally, to further show the performance of KR-LAMPnet-AMP, we adopt least mean square (LMS) to estimate the channel gain based on the result of MUD. With the active users detected, channel estimation can be completed by solving an underdetermined equation, in which the basis matrix $\boldsymbol{\Phi}_{\text {new }}$ is composed of columns in $\boldsymbol{\Phi}$ corresponding to the selected users.

$$
\mathbf{Y}=\boldsymbol{\Phi}_{\text {new }} \mathbf{x}_{\text {new }},
$$


where $\mathbf{x}_{\text {new }}$ is composed of the channel gain of the active users, equivalent to the non-zero entries in $\mathbf{x}$. LMS is applied here

$$
\hat{\mathbf{x}}_{\text {new }}=\left(\boldsymbol{\Phi}_{\text {new }}^{T} \boldsymbol{\Phi}_{\text {new }}\right)^{-1} \boldsymbol{\Phi}_{\text {new }}^{T} \mathbf{Y} .
$$

$\hat{\mathbf{x}}_{\text {new }}$ corresponds to the non-zero entries in $\mathbf{x}$. Thus the channel estimation is done through LMS and the result of MUD.

\section{Simulation Results and Analysis}

To verify the effectiveness of LAMPnet based AMP, we simulate MUD algorithms including KR-BPDN-AMP, OMP-MMV and KR-LAMPnet-AMP. The prefix KR means that Khatri-Rao product is used in the algorithm to transfer the original MUD problem into a SMV problem. The parameters of the tests corresponding to the figures are showm in Table 3.

Table 3. Parameter of the figures.

\begin{tabular}{ccccccc}
\hline & N & L & M & D & K & SNR \\
\hline Figure 4a & 256 & 30 & 15 & 50 & $10 / 20 / 30$ & $10 \mathrm{~dB}$ \\
Figure 4b & 256 & 30 & 15 & 50 & $10 / 20 / 30$ & $10 \mathrm{~dB}$ \\
Figure 5a & 256 & 30 & $15 / 18 / 21$ & 50 & 10 & $10 \mathrm{~dB}$ \\
Figure 5b & 256 & $24 / 27 / 30$ & 15 & 50 & 10 & $10 \mathrm{~dB}$ \\
Figure 6a & 256 & 30 & 15 & $10 / 20 / 50$ & 10 & $10 \mathrm{~dB}$ \\
Figure 6b & 256 & 30 & 15 & 50 & 10 & $10 / 15 / 20 \mathrm{~dB}$ \\
Figure 7 & 256 & 30 & 15 & 50 & $10 / 20$ & $10 \mathrm{~dB}$ \\
\hline
\end{tabular}

Figure 4 shows the ROC curve of KR-BPDN-AMP, OMP-MMV and KR-LAMPnet-AMP with different $K$. It is done when $N=256, D=50, L=30, M=15$, SNR $=10 \mathrm{~dB}$. When the detection probability of BPDN-AMP is over a threshold, even little promotion in detection probability is made at the expense of a substantial rise of false alarm probability. Comparitively, the rise of false alarm probability of KR-LAMPnet-AMP is much smoother and steadier. MMV-OMP performs the best when $K=10$, but worse than KR-LAMPnet-AMP when $K=20,30$. The performance of OMP algorithm is seriously affected by the number of active users since the correlations between pilots of different users are not zero. The influence of the non-zero correlation becomes more serious with the increase of active users. According to the simulation, the weakening of OMP-MMV is the most obvious among KR-BPDN-AMP, OMP-MMV and KR-LAMPnet-AMP. Comprehensively, the detection performance of LAMPnet based AMP outperforms KR-BPDN-AMP and OMP-MMV, especially when the required detection probability is high.

With $M$ larger, $\left(\operatorname{vec}\left(\frac{1}{M} \sum_{i=1}^{M} \mathbf{y}_{i} \mathbf{y}_{i}^{H}\right)\right)$ gets closer to $\mathbf{R}$ and the estimation is more accurate. Figure $5 \mathrm{a}$ shows the ROC curve with different number of antennas. $K$ is set as 10 and other parameters remain unchanged. Figure $5 \mathrm{~b}$ is done with different $L$. Both figures prove that the detection ability of KR-LAMPnet-AMP is better than KR-BPDN-AMP when the required detection ability is high.

Figure $6 \mathrm{a}$ is done with $S N R=10 \mathrm{~dB}$ and other parameters unchanged. The increase in the number of used nets brings better performance but the computational complexity rises meanwhile. Figure $6 \mathrm{~b}$ shows the performance under different signal to noise ratio (SNR) and the superiority of KR-LAMPnet-AMP is clear. 


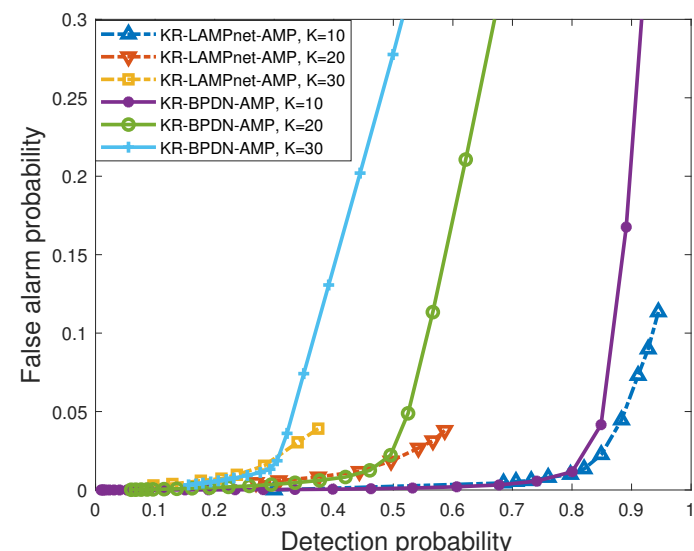

(a) The ROC curve of KR-LAMPnet-AMP and KR-BPDN-AMP

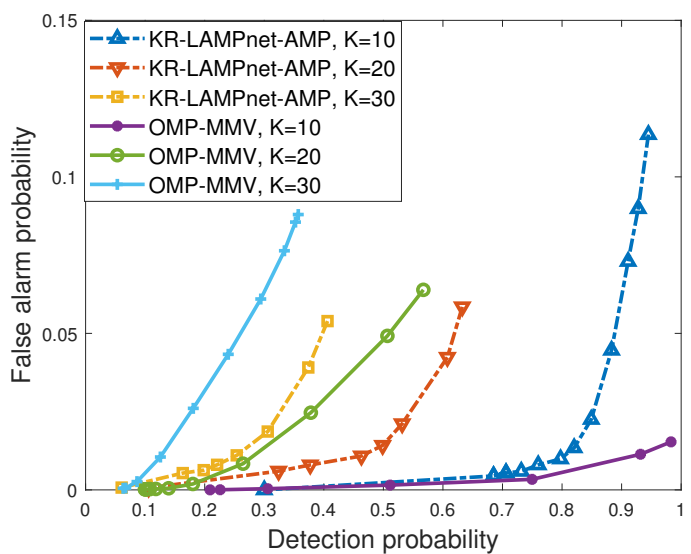

(b) The ROC curve of KR-LAMPnet-AMP and MMV-OMP

Figure 4. Comparision of KR-basis pursuit de-noising approximate message passing (BPDN-AMP), orthogonal matching pursuit (OMP)-multiple measurement vector (MMV) and KR-learning AMP network (LAMPnet)-AMP.

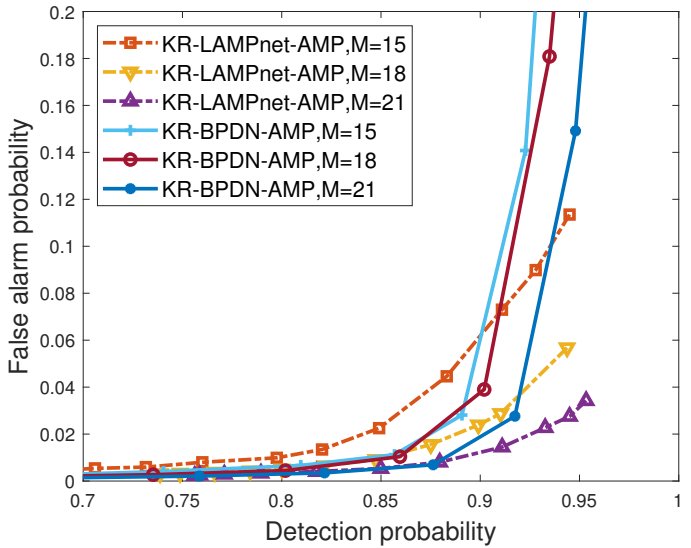

(a) The ROC curve with $M$ varying

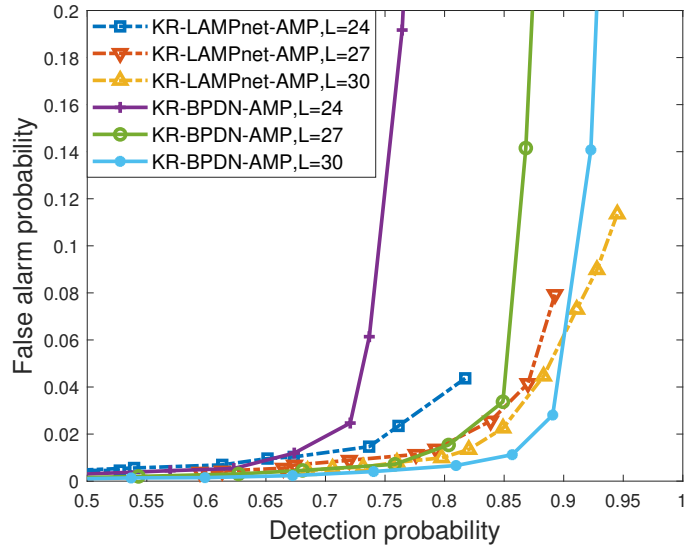

(b) The ROC curve with $L$ varying

Figure 5. Comparision of KR-LAMPnet-AMP and KR-BPDN-AMP.

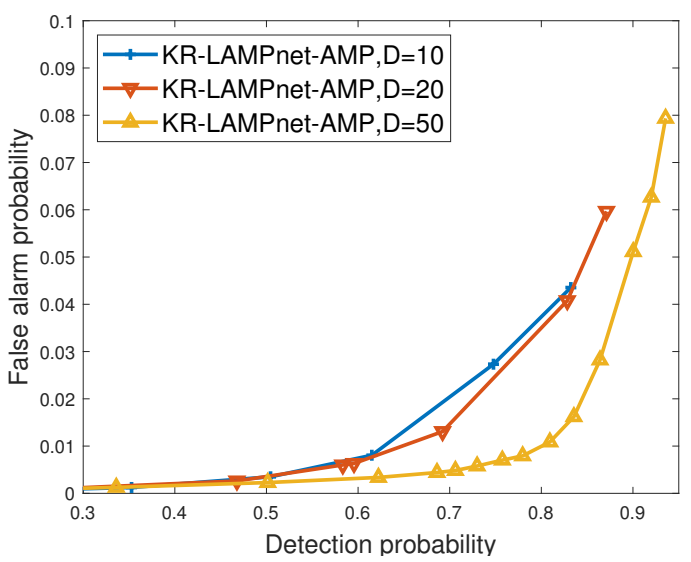

(a) The ROC curve with $D$ varying

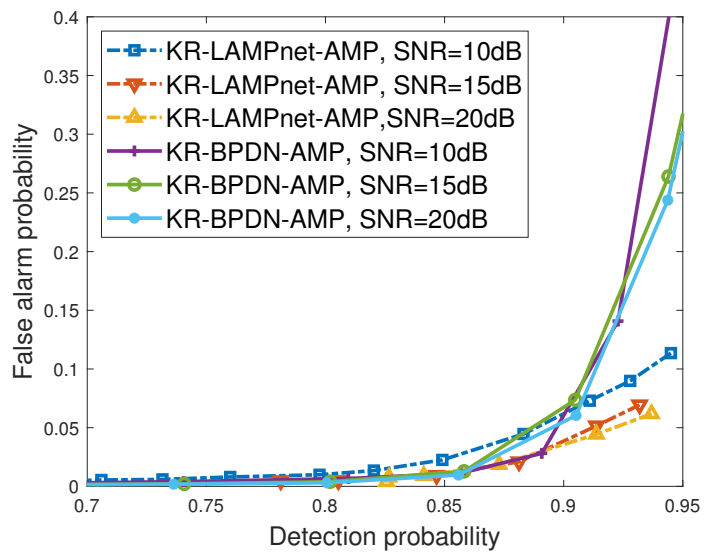

(b) The comparision between KR-LAMPnet-AMP and MMV-OMP

Figure 6. Comparision of KR-LAMPnet-AMP and KR-BPDN-AMP. 
Figure 7 shows the relation between SNR and Root Mean Square Error (RMSE) of the estimated channel between an antenna at the base station and the users, which is recovered with LMS based on the result of MUD. It is done when $N=256, D=50, L=30, M=15$. Obviously, KR-LAMPnet-AMP outperforms KR-BPDN-AMP on the estimation of channel gains and the result verifies the advantage of KR-LAMPnet-AMP.

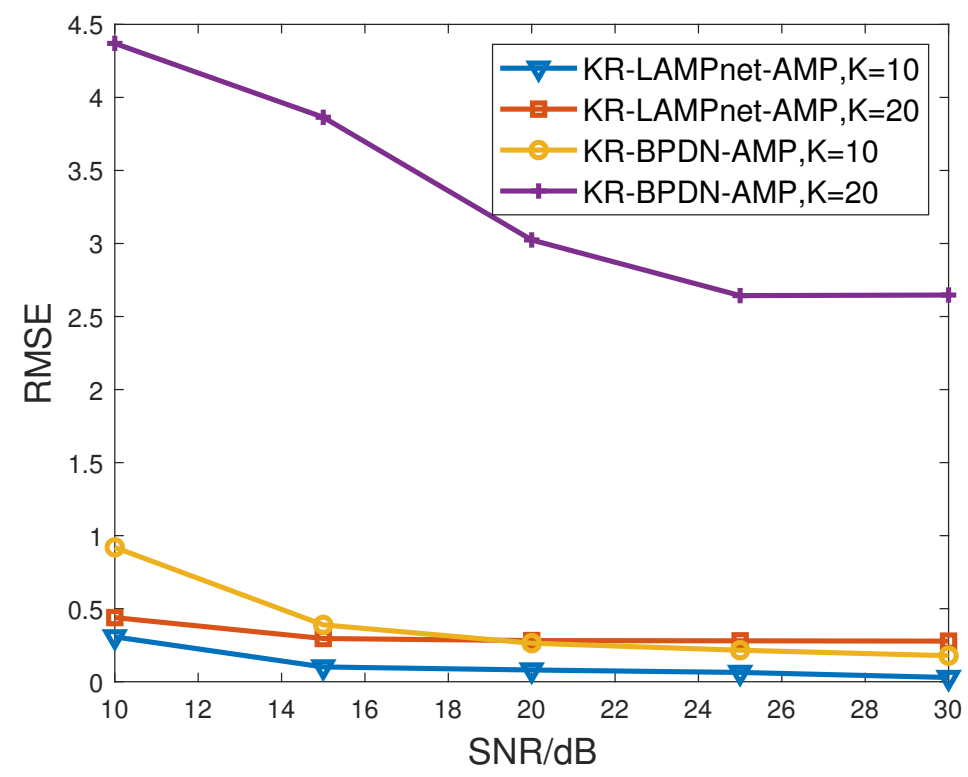

Figure 7. RMSE of the estimated channel.

\section{Conclusions}

In this paper, we have proved that the MUD problem with multiple antennas can be transformed into a SMV problem through applying Khatri-Rao product in the mMTC model. A novel LAMPnet based AMP has been proposed to estimate active users with low error rate and the simulation proves that when the required detection probability is high, LAMPnet based AMP reaches a lower false alarm probability than BPDN-AMP with the detection probability the same. When the number of active users is large, LAMPnet-AMP also outperforms MMV-OMP. The advantage of LAMPnet-AMP mainly concentrates in the lower false alarm probability with high detection probability compared with BPDN-AMP. When the requirement of the detection probability is not hard, LAMPnet-AMP does not outperform BPDN-AMP. Therefore, LAMPnet-AMP is suitable for the conditions with high required detection probability and tolerance for high complexity, which is brought by multiple LAMPnets used in the algorithm. Besides, the threshold of the hard threshold function requires dynamically adjusting according to different circumstances. When the channel environments are noiseless, setting the threshold as the average absolute value of the non-zero entries will exclude active users. Further optimization on the algorithm will follow.

Author Contributions: Methodology, M.S.; software, M.S.; writing, M.S.; validation, P.C.; review and editing, P.C. Both authors have read and agreed to the published version of the manuscript.

Funding: This work was supported in part by the foundation of Shannxi Key Laboratory of Integrated and Intelligent Navigation grant number SKLIIN-20190204, the Pre-research fund of Equipments of China, and the National Natural Science Foundation of China grant number 61801112.

Conflicts of Interest: The authors declare no conflict of interest. 


\section{References}

1. Popovski, P.; Trillingsgaard, K.F.; Simeone, O.; Durisi, G. 5G wireless network slicing for eMBB, URLLC, and mMTC: A communication-theoretic view. IEEE Access 2018, 6, 55765-55779. [CrossRef]

2. Bockelmann, C.; Pratas, N.K.; Wunder, G.; Saur, S.; Navarro, M.; Gregoratti, D.; Vivier, G.; De Carvalho, E.; Ji, Y.; Stefanović, Č.; et al. Towards massive connectivity support for scalable mMTC communications in 5G networks. IEEE Access 2018, 6, 28969-28992. [CrossRef]

3. Dai, L.; Wang, B.; Yuan, Y.; Han, S.; Chih-Lin, C.; Wang, Z. Non-orthogonal multiple access for 5G: Solutions, challenges, opportunities, and future research trends. IEEE Commun. Mag. 2015, 53, 74-81. [CrossRef]

4. Ksairi, N.; Debbah, M. Uplink pilots for multiuser MIMO with mixed grant free and grant based transmissions. In Proceedings of the 2018 IEEE 87th Vehicular Technology Conference, Porto, Portugal, 3-6 June 2018; pp. 1-6.

5. Saito, Y.; Kishiyama, Y.; Benjebbour, A.; Nakamura, T.; Li, A.; Higuchi, K. Non-orthogonal multiple access (NOMA) for cellular future radio access. In Proceedings of the IEEE 77th Vehicular Technology Conference, Dresden, Germany, 2-5 June 2013; pp. 1-5.

6. Ding, Z.; Yang, Z.; Fan, P.; Poor, H.V. On the performance of non-orthogonal multiple access in 5G systems with randomly deployed users. IEEE Signal Process. Lett. 2014, 21, 1501-1505. [CrossRef]

7. Ding, Z.; Fan, P.; Poor, H.V. Impact of user pairing on 5G nonorthogonal multiple-access downlink transmissions. IEEE Trans. Veh. Technol. 2016, 65, 6010-6023. [CrossRef]

8. Wang, B.; Dai, L.; Zhang, Y.; Mir, T.; Li, J. Dynamic compressive sensing based multi-user detection for uplink grant-free NOMA. IEEE Commun. Lett. 2016, 20, 2320-2323. [CrossRef]

9. Wu, B.; Wang, Q. New sub-optimal multiuser detectors for synchronous CDMA systems. In Proceedings of the IEEE Pacific Rim Conference on Communications, Computers, and Signal Processing, Victoria, BC, Canada, 17-19 May 1995; pp. 445-448.

10. Jiang, Y. Sub-optimal multiuser detector using a wavelet chaotic simulated annealing neural network. In Proceedings of the Fourth International Conference on Natural Computation, Jinan, China, 18-20 October 2008; pp. 358-362.

11. Jiang, Y.; Zhong, Z.; Yang, J.; Zhang, M. Sub-optimal multiuser detector using a time-varying gain chaotic simulated annealing neural network. In Proceedings of the Third International Conference on Natural Computation, Haikou, China, 24-27 August 2007; pp. 305-309.

12. Wang, B.; Dai, L.; Yuan, Y.; Wang, Z. Compressive sensing based multi-user detection for uplink grant-free non-orthogonal multiple access. In Proceedings of the IEEE 82nd Vehicular Technology Conference, Boston, MA, USA, 6-9 September 2015; pp. 1-5.

13. Tropp, J.A.; Gilbert, A.C. Signal recovery from random measurements via orthogonal matching pursuit. IEEE Trans. Inf. Theory 2007, 53, 4655-4666. [CrossRef]

14. Donoho, D.L.; Tsaig, Y.; Drori, I.; Starck, J. Sparse solution of underdetermined systems of linear equations by stagewise orthogonal matching pursuit. IEEE Trans. Inf. Theory 2012, 58, 1094-1121. [CrossRef]

15. Davenport, M.A.; Wakin, M.B. Analysis of orthogonal matching pursuit using the restricted isometry property. IEEE Trans. Inf. Theory 2010, 56, 4395-4401. [CrossRef]

16. Schepker, H.F.; Bockelmann, C.; Dekorsy, A. Exploiting sparsity in channel and data estimation for sporadic multi-user communication. In Proceedings of the Tenth International Symposium on Wireless Communication Systems, Ilmenau, Germany, 27-30 August 2013; pp. 1-5.

17. Oyerinde, O.O. Compressive sensing algorithms for multiuser detection in uplink grant free NOMA systems. In Proceedings of the IEEE 89th Vehicular Technology Conference, Kuala Lumpur, Malaysia, 28 April-1 May 2019; pp. 1-6.

18. Chen, D.; Tian, L.; Xu, C. MMV based OMP for DOA estimation with 1-bit measurement. J. Phys. Conf. Ser. 2020, 1550, 032150. [CrossRef]

19. Chen, Z.; Yu, W. Massive device activity detection by approximate message passing. In Proceedings of the IEEE International Conference on Acoustics, Speech and Signal Processing, New Orleans, LA, USA, 5-9 March 2017; pp. 3514-3518.

20. Chen, Z.; Sohrabi, F.; Yu, W. Sparse activity detection for massive connectivity. IEEE Trans. Signal Process. 2018, 66, 1890-1904. [CrossRef] 
21. Xie, R.; Yin, H.; Chen, X.; Wang, Z. Many access for small packets based on precoding and sparsity-aware recovery. IEEE Trans. Commun. 2016, 64, 4680-4694. [CrossRef]

22. Donoho, D.L.; Maleki, A.; Montanari, A. Message passing algorithms for compressed sensing: I. motivation and construction. In Proceedings of the IEEE Information Theory Workshop on Information Theory, Cairo, Egypt, 6-8 January 2010; pp. 1-5.

23. Donoho, D.L.; Maleki, A.; Montanari, A. Message passing algorithms for compressed sensing: II. analysis and validation. In Proceedings of the IEEE Information Theory Workshop on Information Theory, Cairo, Egypt, 6-8 January 2010; pp. 1-5.

24. Perelli, A.; Davies, M.E. Compressive computed tomography image reconstruction with denoising message passing algorithms. In Proceedings of the 23rd European Signal Processing Conference, Nice, France, 31 August-4 September 2015; pp. 2806-2810.

25. Hannak, G.; Perelli, A.; Goertz, N.; Matz, G.; Davies, M.E. Performance analysis of approximate message passing for distributed compressed sensing. IEEE J. Sel. Top. Signal Process. 2018, 12, 857-870. [CrossRef]

26. Teng, C.; Liao, C.; Cheng, H.; Wu, A.A. Reliable compressive sensing (CS) based multi-user detection with power based Zadoff-Chu sequence design. In Proceedings of the IEEE International Workshop on Signal Processing Systems, Lorient, France, 3-5 October 2017; pp. 1-5.

27. Candès, E.J.; Tao, T. Decoding by linear programming. IEEE Trans. Inf. Theory 2005, 51, 4203-4215.

28. Donoho, D.L.; Elad, M.; Temlyakov, V.N. Stable recovery of sparse overcomplete representations in the presence of noise. IEEE Trans. Inf. Theory 2006, 52, 6-18. [CrossRef]

29. Buades, A.; Coll, B.; Morel, J.-M. A review of image denoising algorithms, with a new one. Siam J. Multiscale Model. Simul. 2005, 4, 490-530. [CrossRef]

30. Portilla, J.; Strela, V.; Wainwright, M.J.; Simoncelli, E.P. Image denoising using scale mixtures of Gaussians in the wavelet domain. IEEE Trans. Image Process. 2003, 12, 1338-1351. [CrossRef] [PubMed]

31. Dabov, K.; Foi, A.; Katkovnik, V.; Egiazarian, K. Image denoising by sparse 3-D transform-domain collaborative filtering. IEEE Trans. Image Process. 2007, 16, 2080-2095. [CrossRef] [PubMed]

(C) 2020 by the authors. Licensee MDPI, Basel, Switzerland. This article is an open access article distributed under the terms and conditions of the Creative Commons Attribution (CC BY) license (http:/ / creativecommons.org/licenses/by/4.0/). 